Char act eri stics of pl asm par ameters and turbul ence in the i sot ope-ming and the non- mixing st at es in hydrogen deuteri um mixture pl asmas in the I arge hel i cal device

\begin{tabular}{|l|l|}
\hline $\begin{array}{l}\text { j our nal or } \\
\text { publ i cat i on } \mathrm{ti} \text { t l e }\end{array}$ & Nucl ear Fusi on \\
\hline vol une & 61 \\
\hline number & 1 \\
\hline page r ange & 016012 \\
\hline year & $2020-11-23$ \\
\hline NAl S & 12443 \\
\hline URL & ht t p: //hdl . handl e. net /10655/00012546 \\
\hline
\end{tabular}




\title{
Characteristics of plasma parameters and turbulence in the isotope-mixing and the non-mixing states in hydrogen-deuterium mixture plasmas in the Large Helical Device
}

\author{
K.Ida, ${ }^{1,2}$ M.Yoshinuma, ${ }^{1,2}$ K.Tanaka, ${ }^{1}$ M.Nakata,,${ }^{1,2}$ T.Kobayashi, ${ }^{1,2}$ Y.Fujiwara, ${ }^{1}$ \\ R.Sakamoto, ${ }^{1,2}$ G.Motojima, ${ }^{1}$ S.Masuzaki, ${ }^{1,2}$ and the LHD Experiment Group ${ }^{1}$ \\ ${ }^{1}$ National Institute for Fusion Science, National Institutes of Natural Sciences, Toki, Gifu 509-5292, Japan \\ ${ }^{2}$ The Graduate University for Advanced Studies, SOKENDAI, Toki, Gifu 509-5292, Japan
}

(Dated: October 16, 2020)

\begin{abstract}
Characteristics of plasma parameters and turbulence in the isotope-mixing and the non-mixing states in hydrogen-deuterium mixture plasmas in the Large Helical Device are discussed. The isotope mixing state is characterized by the uniform isotope ratio profile regardless of the location of the particle source of each species in the isotope mixture plasma. The isotope non-mixing state is identified by the non-uniform isotope ratio profile measured with bulk charge exchange spectroscopy when the beam fueling isotope species differs from the recycling isotope species. The effect of collisionality, $T_{e} / T_{i}$ ratio, sign of density gradient on transition between isotope mixing and nonmixing is discussed. The plasma parameters preferable for the non-mixing state are found to be lower collisionality, higher $T_{e} / T_{i}$, and negative or zero density gradient (peaked or flat density profile). The time scale of transition from non-mixing to mixing is evaluated by the hydrogen and deuterium pellet injection near the plasma edge and is found to be less than $5 \mathrm{~ms}$, which is much shorter than the particle confinement time. The strong correlation between isotope mixing and turbulence characteristics is observed. This strong correlation suggests the change in turbulence is a strong candidate for the mechanism causing the transition between uniform and non-uniform isotope density ratio profiles.
\end{abstract}

PACS numbers:

\section{INTRODUCTION}

Isotope mixing is a new bifurcation phenomena [1] in the mixture plasma which can not be explained solely by the conventional diffusive/non-diffusive transport in single-ion species plasma. In the conventional diffusive/non-diffusive model, each isotope has its own diffusion coefficient (D) and pinch velocity (V). These coefficients between different isotope species (for example, hydrogen and deuterium) have been considered to depend on the local parameter such as electron density, temperature, magnetic shear, and radial profile of each isotope species is determined independently. However, recent simulation and experiment reveals that the radial profile of each isotope species, for example, hydrogen $(\mathrm{H})$ and deuterium (D), is determined by the complex transport processes with the strong coupling among the different particle species, rather than the individual diffusion coefficients $\left(D_{\mathrm{H}}\right.$ and $\left.D_{\mathrm{D}}\right)$ and pinch velocities $\left(V_{\mathrm{H}}\right.$ and $\left.V_{\mathrm{D}}\right)$ between two species.

In single-ion species plasma, the electron density $n_{e}$ is equal to ion density $n_{i}$, and the electron and ion diffusion coefficients are identical due to the quasi-neutral condition. However, in the isotope mixture plasma (hydrogen and deuterium mixture plasma) the ratio of ion diffusion coefficient to electron diffusion coefficient $D_{i} / D_{e}$ (e.g., $\left.D_{H} / D_{e}, D_{D} / D_{e}\right)$ has a strong impact on each isotope density profile. Because the hydrogen and deuterium pellet injection experiment in LHD demonstrated that there is no significant difference in particle transport between hydrogen and deuterium [2], the hydrogen diffusion coefficient is assumed to be equal to the deuterium diffusion coefficient as $D_{H}=D_{D}=D_{i}$. The transport simulation model (QuaLiKiz $[3,4]$ ) using nonlinear gyrokinetic simulation code (GKW [5]) predicts that the ratio $D_{i} / D_{e}$ strongly depends on the mode of turbulence [6]. The electron diffusion coefficient is much larger than that of ions $\left(D_{e} / D_{i} \gg 1\right)$ when the trapped electron mode (TEM) $[7,8]$ is dominant. In this case, each isotope can have a different profile because only the sum of the density of isotope species is required to be balanced to the electron density profile determined by a large electron diffusion to satisfy the quasi-charge neutrality condition. The isotope density ratio profile varies significantly depending on the location of the source of each isotope species for the given electron density profile. For example, the hydrogen density profile can be significantly peaked by the hydrogen beam fueling in the hydrogen deuterium mixture plasma with the condition of deuterium dominant recycling. In contrast, the ion diffusion coefficient becomes much larger than that of electron $\left(D_{i} / D_{e} \gg 1\right)$ when the ion temperature gradient (ITG) mode $[9,10]$ is dominant. In this case, the radial distribution of isotope ratio (for example, the ratio of hydrogen density to deuterium density in the isotope mixture plasma) becomes uniform regardless of the source location of each isotope species because of the large ion diffusion. This is essential transport process in multiple-ion-species plasmas, and leads to isotope mixing state or non-mixing state, regardless of the almost identical shape of the electron density profile. 
Therefore, there are two states of isotope distribution predicted in the isotope mixture plasma. One is isotope mixing state which is characterized by the uniform isotope density ratio profile regardless of the source location of each isotope species. The other is isotope non-mixing state which is characterized by the non-uniform isotope density ratio profile if the isotope species of core beam fueling differs from the isotope species of edge recycling. The transition between the two states was reported in the isotope mixture experiment in Large Helical Device (LHD) [11], where gyrokinetic simulation by GKV indicates the change of the dominant microinstability between TEM and ITG [12-16]. However, more experimental and theoretical study is necessary to understand the physics mechanism for isotope mixing. The parameter dependence of the transition and the relation to the turbulence characteristics have not been investigated, although these studies are an important issue for testing the hypothesis of isotope mixing/non-mixing profile formation by turbulence mode.

Although the turbulence characteristics in the ECH, NBI, and ion-ITB plasma have been reported in LHD [17-20], the characteristics of turbulence in the isotope non-mixing and mixing plasma has not been studied in detail due to the lack of measurements of radial profiles of isotope density. In this paper, parameter dependence and the relation between turbulence characteristics and isotope mixing or non-mixing are discussed. The isotope mixing and non-mixing states are evaluated by the radial profile of hydrogen to deuterium density ratio measured with bulk charge exchange spectroscopy [21-24], while the turbulence characteristics are investigated by the electron density fluctuation spectrum measured with two-dimensional phase contrast imaging (2D-PCI).

\section{TURBULENCE CHARACTERISTICS IN ISOTOPE MIXING AND NON-MIXING PLASMAS}

It should be noted that the isotope mixing/non-mixing is determined by the ratio of anomalous ion diffusion coefficient to anomalous electron diffusion coefficient $\left(D_{i} / D_{e}\right)$ in turbulent transport. This is in contrast to the ion/electron root in helical plasmas, where the sign of radial electric field $\left(E_{r}\right)$ is determined by the ratio of neoclassical ion diffusion coefficient to neoclassical electron diffusion coefficient $\left(D_{i}^{N C} / D_{e}^{N C}\right)$ for $E_{r}$ $=0$. This is because electron and ion diffusion coefficients strongly depend on $E_{r}$ in neoclassical transport but they are independent of $E_{r}$ in turbulent transport. Therefore, $D_{i} / D_{e}>1$ corresponds to the mixing state, while $D_{i} / D_{e}<1$ corresponds to the non-mixing state in turbulent transport. In neoclassical transport, $D_{i}^{N C} / D_{e}^{N C}>1$ corresponds to ion root $\left(E_{r}<0\right)$, while $D_{i}^{N C} / D_{e}^{N C}<1$ corresponds to electron root $\left(E_{r}>0\right)$. In the standard configuration in Large Helical Device (LHD), the anomalous diffusion coefficient is much larger than the neoclassical diffusion coefficient $\left(D_{i} / D_{i}^{N C} \gg 1\right.$ and $\left.D_{e} / D_{e}^{N C} \gg 1\right)[25]$. In the parameter regime of this experiment, TEM turbulence and ITG turbulence are the most likely turbulence mode in the ion gyroradius scale. Because the density profile is flat or hollow, the TEM turbulence is predicted to be destabilized near the plasma edge where the density gradient is negative and TEM or ITG turbulences are predicted to be more pronouncedly destabilized in the core region where the density gradient is close to zero or positive. TEM indicates a higher wavenumber spectrum of the growth rate than ITG mode and mode frequency around $k_{\perp} \rho_{t i} \sim 1$, so that TEM and ITG turbulence are expected to coexist when the profile condition exceeds the criticality for destabilizations in the plasma in LHD. Coexistenece of multi modes (low $k$ ITG, high $k$ ITG, TEM, and ETG) is commonly observed in tokamaks [26].

Since the TEM tends to be stabilized by the electronion collisions[27], the ITG turbulence becomes more dominant in higher collissionality plasma even for the same $R / L_{T_{i}}, R / L_{T_{e}}$, and $R / L_{n_{e}}$, where $R, L_{T_{i}}, L_{T_{e}}$, and $L_{n_{e}}$ are major radius, scale length of ion temperature, electron temperature, and electron density. This is because the growth rate of TEM turbulence sharply increases as the collisionality decreases, while the growth rate of ITG turbulence has weak dependence on collisionality [27]. In order to study the collisionality dependence of isotope mixing/non-mixing and turbulence characteristics, simultaneous measurements for isotope density ratio profile and turbulence have been performed in LHD. In this experiment, the hydrogen neutral beam provides a significant particle source in the plasma core $\left(r_{\text {eff }} / a_{99}<0.4\right)$, while the recycling from the wall and the divertor provides the particle source near the plasma edge. In order to identify the non-mixing state from the isotope ratio profile, ion species of the source near the plasma edge should be different from the ion species of the source in the plasma core. If the ion species of the core source is the same as that of the edge source, a non-mixing state can not be observed even when the plasma is in the nonmixing state. Therefore, the ratio of deuterium influx to hydrogen influx was kept to be as high as possible by wall conditioning with deuterium gas before the experiment and between shots.

Figure 1 shows the radial profiles of electron density, electron temperature, ion temperature, hydrogen and deuterium density, and fraction of hydrogen density $n_{\mathrm{H}} /\left(n_{\mathrm{H}}+n_{\mathrm{D}}\right)$ measured with bulk charge exchange spectroscopy for lower collisionality plasma $\left(\bar{n}_{e}\right.$ $\left.=1.39 \times 10^{19} \mathrm{~m}^{-3}\right)$ and higher collisionality plasma $\left(\bar{n}_{e}=\right.$ $3.85 \times 10^{19} \mathrm{~m}^{-3}$ ), where $\bar{n}_{e}$ is line averaged electron density. Although both electron temperature $\left(T_{e}\right)$ and ion temperature $\left(T_{i}\right)$ of lower collisionality plasma are higher than those of higher collisionality plasma, the temperature ratio of electron to ion $\left(T_{e} / T_{i}\right)$ is comparable for these two discharges. The hydrogen density profile is similar to the deuterium density profile in the isotope mixing state. In the isotope non-mixing state, the hy- 


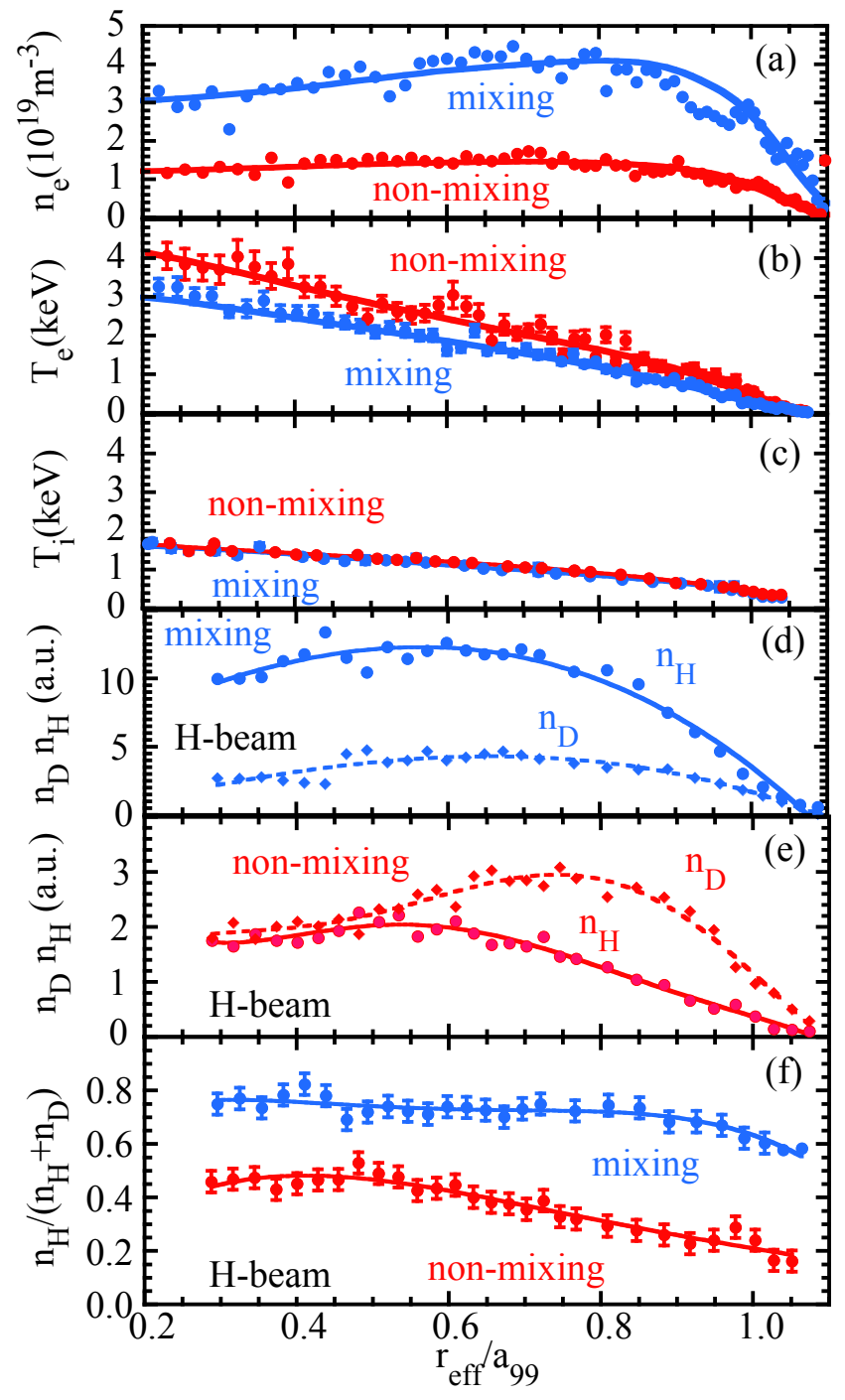

FIG. 1: Radial profiles of (a) electron density, (b) electron temperature, (c) ion temperature, and (d)(e) hydrogen and deuterium density, (f) fraction of hydrogen density $n_{\mathrm{H}} /\left(n_{\mathrm{H}}+n_{\mathrm{D}}\right)$ in the isotope mixing (\#142325 5.23s) and nonmixing (\#142315 3.73s) states in the hydrogen and deuterium mixture plasma with hydrogen beam fueling.

drogen density profile is peaked and deuterium density profile becomes hollow. As seen in the radial profile of the fraction of hydrogen density $n_{\mathrm{H}} /\left(n_{\mathrm{H}}+n_{\mathrm{D}}\right)$, the peaking of hydrogen fraction is clearly observed in the low collisionality plasma. This peaking of hydrogen fraction is due to the combination of beam fueling of hydrogen $(\mathrm{H}) \mathrm{NBI}$ and deuterium (D) dominated recycling $\left(\Gamma_{D} / \Gamma_{H}=4\right)$, which indicates that the plasma is in the non-mixing state. Here, $\Gamma_{D}$ and $\Gamma_{H}$ are the influx (inward radial flux of deuterium neutral and hydrogen neutral) evaluated from $\mathrm{H}_{\alpha}$ and $\mathrm{D}_{\alpha}$ intensity measured with passive spectroscopy. This non-mixing state is an equilibrium steady-state with the balance between the source
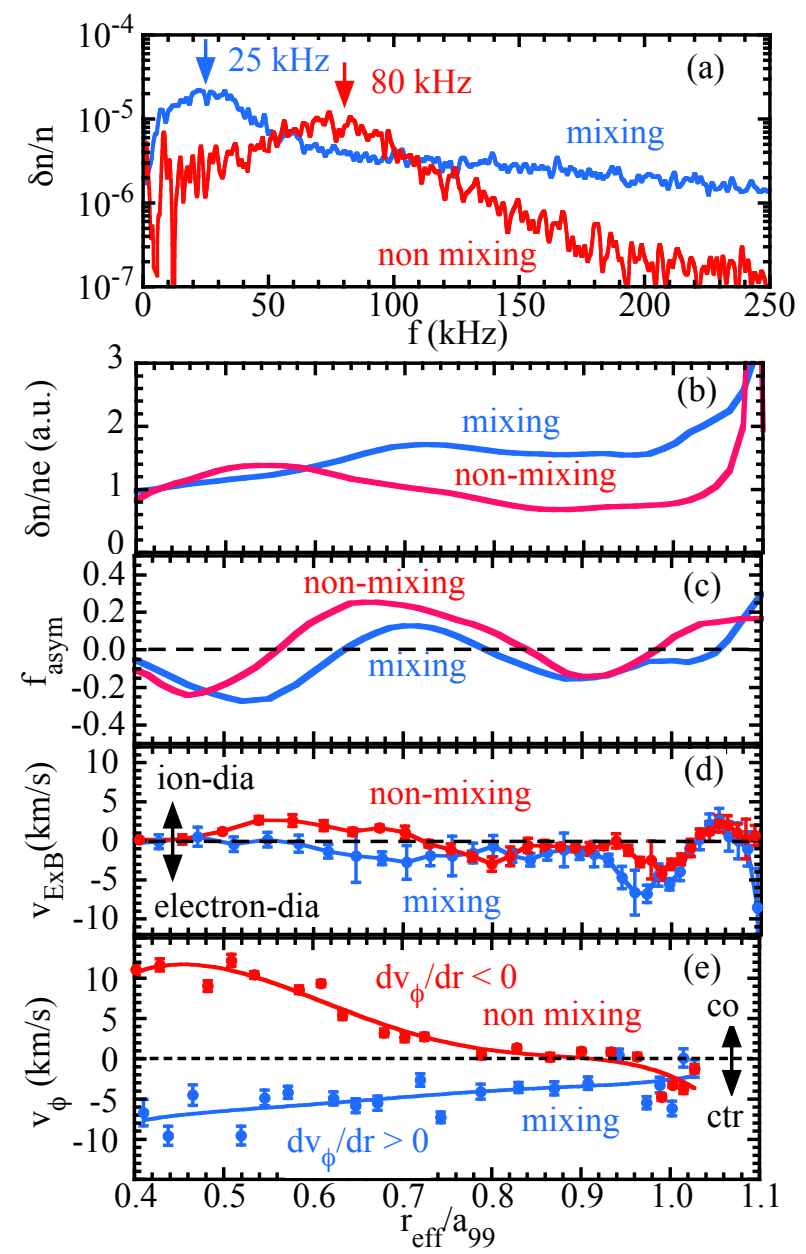

FIG. 2: (a) Density fluctuation spectrum and radial profiles of (b) density fluctuation amplitude, (c) asymmetry factor $f_{\text {asym }}$ of density fluctuation, (d) $E \times B$ velocity, and (e) intrinsic toroidal flow veloicty in the isotope mixing (\#142325 5.23s) and non-mixing (\#142315 3.73s) states in the hydrogen and deuterium mixture plasma with hydrogen beam fueling.

(hydrogen in the core and deuterium near the edge) and the transport. In the mixing state, the hydrogen fraction profile becomes flat except for near the edge in the higher collisionality plasma. The increase of hydrogen fraction is attributable to the increase of hydrogen recycling $\left(\Gamma_{D} / \Gamma_{H}=1.2\right)$ but the flattening of hydrogen fraction profile is due to the isotope mixing.

The clear difference in the turbulence spectrum between these two plasmas is also observed. Density fluctuation spectrum, radial profile of fluctuation amplitude normalized by density and up-down asymmetry factor measured with 2D-PCI is plotted in Figure 2. Here, the up-down asymmetry factor is defined by $f_{\text {asym }}=\left(\delta n^{\text {down }}-\delta n^{\text {up }}\right) /\left(\delta n^{\text {down }}+\delta n^{\text {up }}\right)$. The spectrum is from line integrated 2D-PCI signal, where spatially integrated at $r_{\text {eff }} / a_{99}>0.4,5 k H z<f<500 \mathrm{kHz}$, 
$0.1 \mathrm{~mm}^{-1}<k<0.8 \mathrm{~mm}^{-1}$. 2D-PCI can resolve spatial distribution of turbulence from the propagation direction of turbulence by assuming $k_{\|}=0[28,29]$. However, frequency spectrum from spatially and wave number integrated turbulence shows clear difference in non mixing and mixing state. Here, the background level of PCI diagnostics evaluated from the fluctuation spectrum in the period of no plasma is subtracted. Because the radial profile of $E \times B$ velocity is almost identical between the isotope mixing and the non-mixing plasma, the $E \times B$ Doppler shift is considered to be almost identical between the isotope mixing and the non-mixing plasma.

In the plasma with a non-mixing state, the density fluctuation spectrum has a peak at a higher frequency of $85 \mathrm{kHz}$. The characteristics of density fluctuation with a higher frequency peak observed in LHD resemble the Quasi-coherent (QC) modes, known as a type of the trapped electron mode (TEM) turbulence, namely, QC-TEM. This mode (QC-TEM) has been observed in HL-2A and J-TEXT ohmic heating plasma [30] and in KSTAR ECH plasmas [31]. The characteristic frequencies of a higher frequency peak in LHD are $40-100 \mathrm{kHz}$ with a peak of $80 \mathrm{kHz}$ (see Figure 2(a)), which is the same range of QC-TEM observed in HL-2A and J-TEXT $(30-140 \mathrm{kHz})$. The parameter dependence of appearance/disappearance of the higher frequency peak of density fluctuation observed in LHD is also quite similar to the parameter dependence of QC-TEM, where the QC modes appear in the plasma with lower collisionality or higher $T_{e} / T_{i}$ ratio. The change of the mode from QC-TEM to ITG associated with the transition from the LOC regime to the SOC regime is also predicted from non-linear gyrokinetic simulation [32]. The QC-TEM mode appears in the LOC regime (lower density regime) but it disappears in the SOC regime (higher density regime), where the ITG mode becomes dominant.

In the plasma with the mixing state, this higher frequency peak disappears, and a lower frequency peak appears at $25 \mathrm{kHz}$. The frequency of the peak is consistent with the frequency at the maximum growth rate for ITG turbulence $(f \leq \sim 20 \mathrm{kHz})$ calculated by the GKV code $[11,33]$. The GKV code is a linear gyrokinetic numerical simulation code for multi-ion species. Radial profiles of hydrogen, deuterium, and carbon ions and the fraction of helium ion measured are taken into account in this simulation. The amplitude of fluctuation normalized by density in the region of $r_{\text {eff }} / a_{99}>0.6$ is larger in the mixing state than the non-mixing state and the up-down asymmetry factor $f_{\text {asym }}$ at $r_{\text {eff }} / a_{99}=0.7$ is larger in the isotope non-mixing plasma than in the isotope mixing plasma. The reason for the up-down asymmetry is the difference of the projection of the two vectors $\left(k_{r}\right.$ and $k_{\theta}$ ) of turbulence propagation to PCI measurements [34]. Therefore, the difference in up-down asymmetry factor implies the change in the type of turbulence in these two plasmas.

As seen in Figure 2(e), the gradient of intrinsic toroidal flow $\left(d v_{\phi} / d r\right)$ has opposite sign between these two dis-

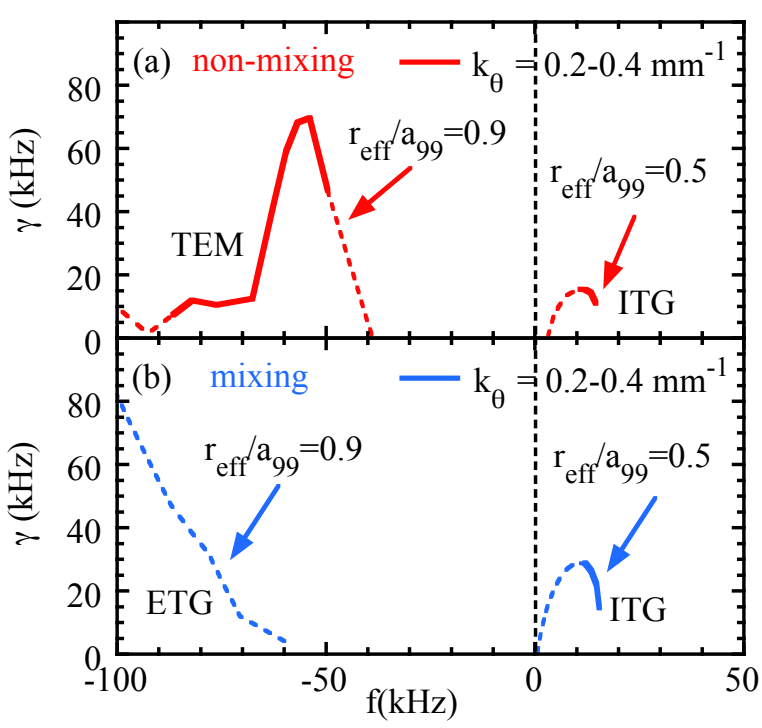

FIG. 3: Linear growth rate at $r_{\text {eff }} / a_{99}=0.5$ and 0.9 for the non-mixing (\#142315) and isotope-mixing (\#142325) states calculated with GKV. The linear growth rates for the wave number of $k_{\theta}=0.2-0.4 \mathrm{~mm}^{-1}$ are indicated with solid line and the dashed lines correspond to the other wave number ( $k_{\theta}<0.2 \mathrm{~mm}^{-1}$ and $\left.k_{\theta}>0.4 \mathrm{~mm}^{-1}\right)$.

charges, where there is no net toroidal torque driven by tangential neutral beams. It is well known that the residual stress produced the gradient of toroidal flow due to the symmetry breaking $\left.\left(k_{\|}^{\text {co }} \neq k_{\|}^{\text {ctr }}\right)\right)$. Because of the boundary condition of $V_{\phi}=0$ at the plasma boundary, this residual stress produced is a strong candidate for intrinsic toroidal flow $[35,36]$. Therefore, the direction of intrinsic toroidal flow depends on the turbulence mode. In LHD, the intrinsic toroidal flow is in the co-direction in the low collisionality regime, while it is in the counterdirection in the higher colllisionality regime [37].

In Alcator C-mode, the TEM mode turbulence is observed when the direction of intrinsic toroidal flow is co-direction and this TEM turbulence disappears associated with the flow reversal from co-direction to counterdirection, which demonstrates that the TEM mode turbulence drives the intrinsic toroidal flow in co-direction. Therefore, the intrinsic toroidal flow in co-direction also suggests the existence of TEM mode turbulence [38]. The characteristics of turbulent spectrum are quite different between isotope mixing and non-mixing plasma. These observations suggest that the change in isotope mixing /non-mixing is attributed to the change in turbulence mode.

Figure 3 shows the linear growth rate at core region $\left(r_{\text {eff }} / a_{99}=0.5\right)$ and at the edge region $\left(r_{\text {eff }} / a_{99}=0.9\right)$ for the non-mixing and isotope-mixing states calculated with GKV $[12,15]$. PCI measurements show that the peak of the wave number of density fluctuation is $\sim 0.3 \mathrm{~m}^{-1}$. 

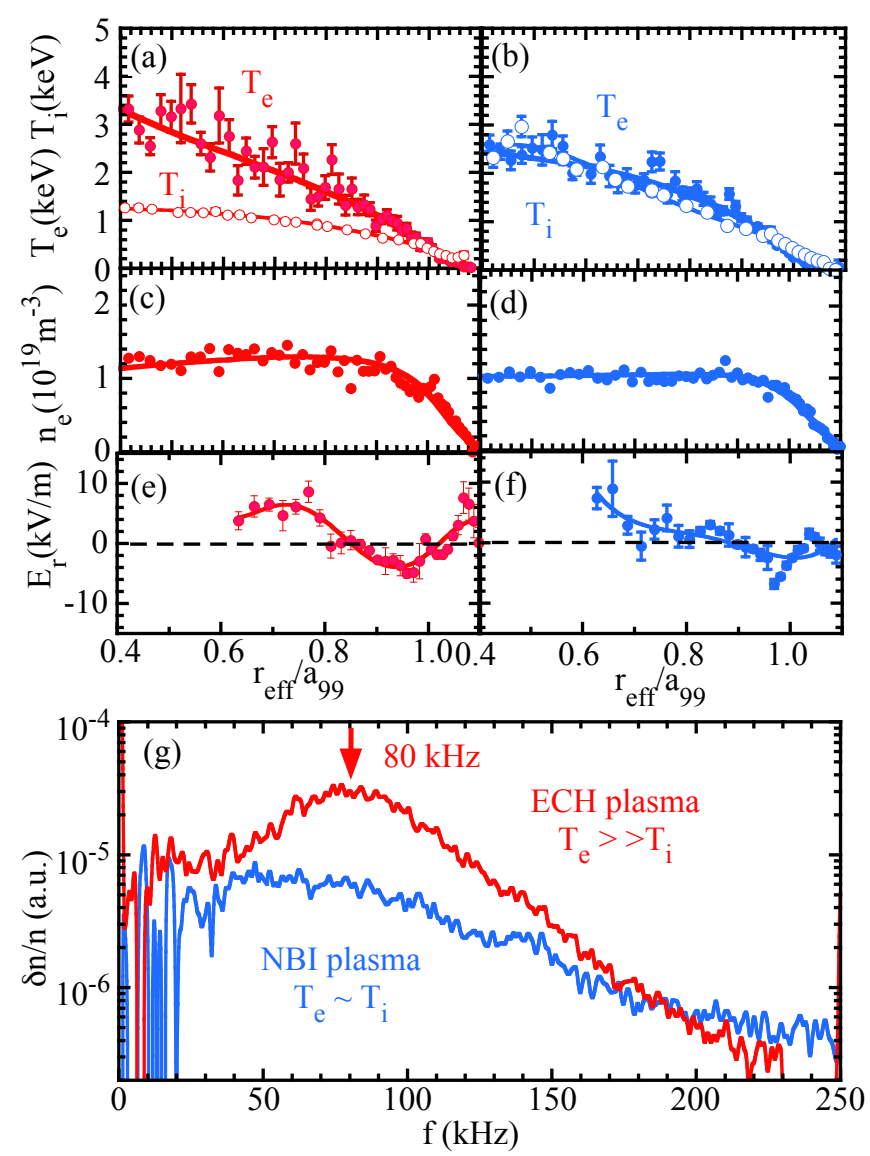

FIG. 4: Radial profiles of (a)(b) electron and ion temperature, (c)(d) electron density, (e)(f) radial electric field, and (g) density fluctuation spectrum in the (a)(c)(e) ECH plasma (\#139080) and (b)(d)(f) NBI deuterium plasma (\#139727).

The linear growth rates for the wave number of $k_{\theta}=0.2$ $-0.4 \mathrm{~mm}^{-1}$ are plotted with a solid line in order to indicate the range for the density fluctuations measured with PCI. In the isotope-mixing states, the TEM is destabilized near the plasma edge where the density and electron temperature gradients are relatively sharp and ITG mode is destabilized at the plasma core where the density gradient is positive (hollow density). In contrast, in the mixing state, the TEM is stabilized near the plasma edge and ITG is still destabilized at the plasma core. In the mixing state, the ETG mode is also destabilized near the plasma edge. Because the wave number for the ETG mode is larger $\left(k>0.4 \mathrm{~mm}^{-1}\right)$ than the wave number for TEM, this mode is not observed in the density fluctuations in PCI measurements.

The comparison of the turbulence spectrum in the ECH plasma and the NBI plasma is important to investigate in the turbulence modes that contribute the turbulence peak at lower frequency and that at higher frequency. This is because $T_{e} / T_{i}$ ratio is quite different between ECH and NBI plasma. TEM turbulence is predicted to be dominant in the low density ECH plasma because of the large normalized electron temperature gradient in the plasma with high $T_{e} / T_{i}$ ratio. In contrast, the ITG turbulence is predicted to be dominant in the NBI plasma where the ion temperature gradient is steeper.

Figure 4 shows the radial profiles of electron and ion temperature, electron density, and radial electric field. The electron temperature is three times higher than the ion temperature in the ECH plasma, while the electron temperature is almost identical to ion temperature in the NBI plasma. The electron density is low $\left(1 \times 10^{19} \mathrm{~m}^{-3}\right)$ and the electron density profile is almost flat. The linear gyrokinetic simulations GKV predicts that the TEM is destabilized because the normalized ion temperature gradient is relatively small in the core $\left(r_{\text {eff }} / a_{99}=0.3\right)$ of the ECH plasma, where the $T_{e} / T_{i}$ ratio is $\sim 3$. It also predicts that the ITG mode is destabilized in the core of the NBI plasma, where the $T_{e} / T_{i}$ ratio is close to unity. Near the plasma edge $\left(r_{\text {eff }} / a_{99}=0.9\right)$, the TEM is predicted to be destabilized due to the negative density gradients [39]. When both ITG mode and TEM are destabilized, the growth rate of TEM often becomes larger than that of ITG mode $\left(\gamma_{\text {TEM }}>\gamma_{\text {ITG }}\right)$ at higher wave number (and higher frequency).

There are no large differences in the radial electric field profile between these ECH and NBI plasmas. Therefore, the Doppler shifts of density fluctuation due to the $E \times B$ drift are similar between these two plasma. However, the density fluctuation spectrum measured with PCI shows the clear difference. The peak at $80 \mathrm{kHz}$ is significant in the ECH plasma, which is similar to the plasma with isotope non-mixing plotted in Figure 2. In the similar ECH plasma, TEM-like fluctuation characteristics are observed in the PCI measurements [39]. In the NBI plasma, the peak at $80 \mathrm{kHz}$ disappears completely, which suggests the reduction of TEM turbulence in the plasma core. These observations imply the strong linkage between the transition from non-mixing to mixing and the change in turbulence characteristics.

\section{IMPACT OF DENSITY GRADIENT TO ISOTOPE MIXING AND NON-MIXING}

The density gradient is also an important parameter for the transition from TEM turbulence to ITG turbulence temperature gradient [39]. In order to investigate the impact of the density gradient on the transition from non-mixing to mixing, two discharges with similar line averaged density and temperature are compared. Figure 5 displays the time evolution of line averaged electron density, density fluctuation spectrum, the ratio of density fluctuation amplitude integrated in the range of $40-90 \mathrm{kHz}$ to that of $4-40 \mathrm{kHz}$, and the fraction of hydrogen density $n_{\mathrm{H}} /\left(n_{\mathrm{H}}+n_{\mathrm{D}}\right)$ at $r_{\text {eff }} / a_{99}=0.68$ and 0.96 . Schemes of heating are identical for the two discharges 


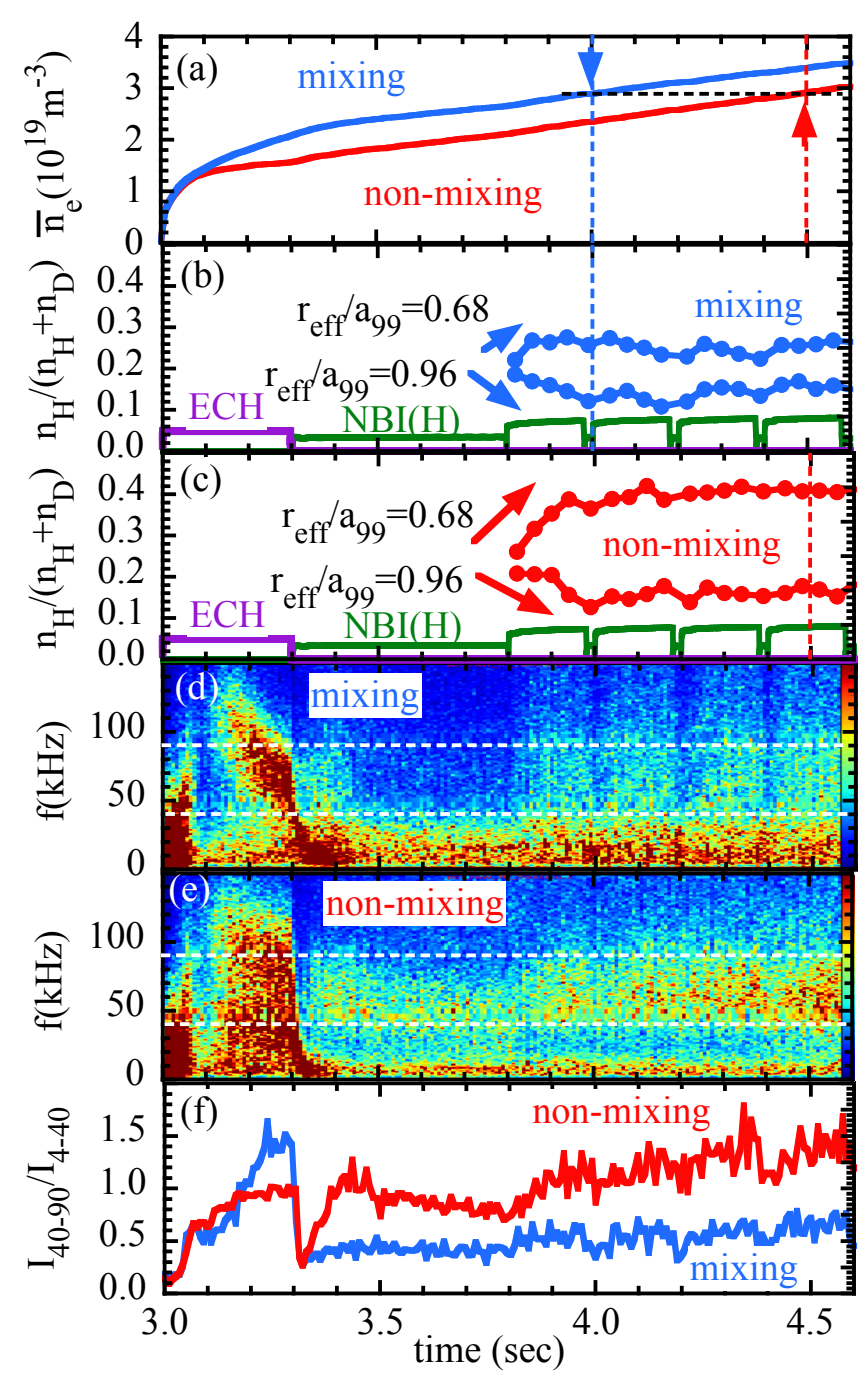

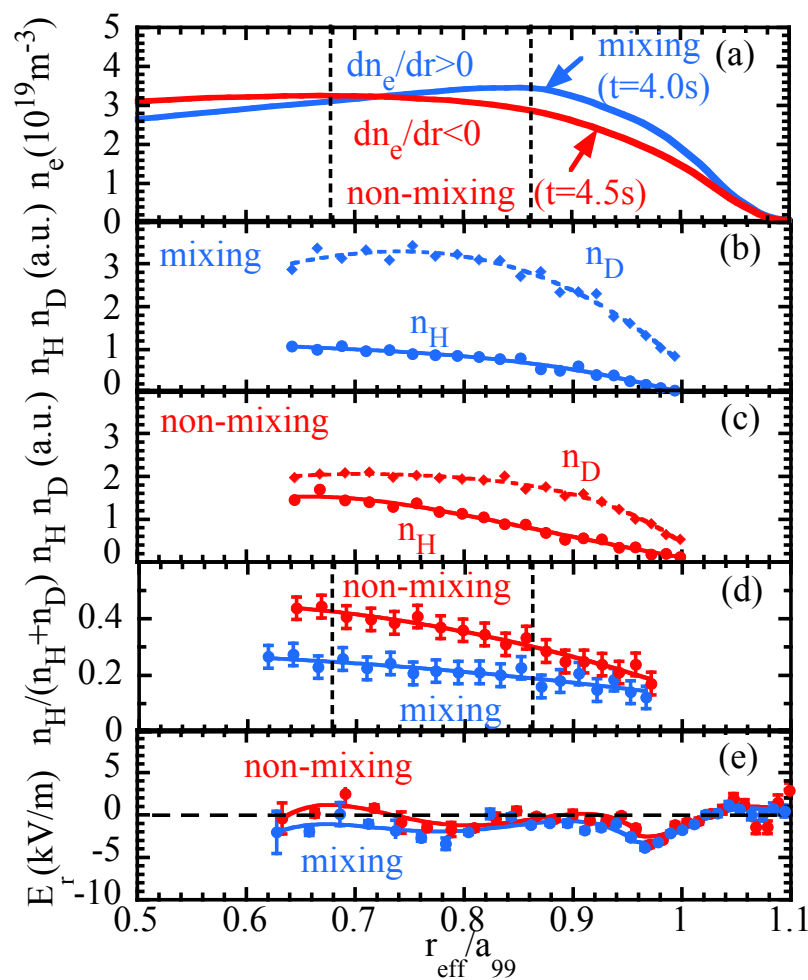

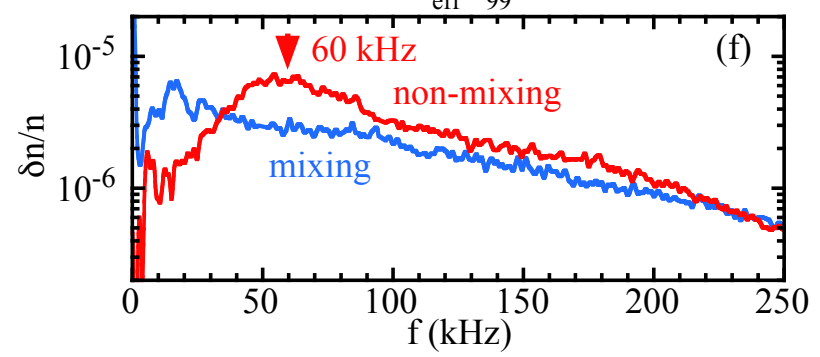

FIG. 5: Time evolution of (a) line averaged electron density, (b)(c) the fraction of hydrogen density $n_{\mathrm{H}} /\left(n_{\mathrm{H}}+n_{\mathrm{D}}\right)$ at $r_{\text {eff }} / a_{99}=0.68$ and 0.96 , respectively, (d)(e) density fluctuation spectrum, and (f) the ratio of density fluctuation amplitude integrated in the range of $40-90 \mathrm{kHz}$ to that of $4-40 \mathrm{kHz}$, for mixing (\#158390) and non-mixing discharge (\#159396) in the hydrogen and deuterium mixture plasma with hydrogen beam fueling.

plotted. The plasma is initiated by ECH for $t=3.0-3.3$ secs, followed by hydrogen neutral beam. The power of hydrogen neutral beam increases from $t=3.8 \mathrm{sec}$. The difference between these discharges is the amount of recycling. The density increases faster in the discharger with higher recycling and the density increases slower in the discharger with lower recycling. The isotope non-mixing state is observed in the discharge with lower recycling, where the hydrogen fraction in the core $\left(r_{\text {eff }} / a_{99}=0.68\right)$ increases rapidly after the increase of NBI power due to the core beam fueling by hydrogen NBI. The increase
FIG. 6: Radial proles of (a) electron density, (b)(c) hydrogen and deuterium density, (d) fraction of hydrogen density $n_{\mathrm{H}} /\left(n_{\mathrm{H}}+n_{\mathrm{D}}\right)$, (e) radial electric field, and (f) density fluctuation spectrum at $t=4.0 \mathrm{~s}$ in the isotope mixing plasma (\#158390) and at $t=4.5 \mathrm{~s}$ in the non-mixing plasma (\#159396) plotted in figure 3 .

of the hydrogen fraction saturates in $0.2 \mathrm{sec}$, which is the order of particle confinement time in the plasma core region. In contrast, isotope mixing (but not complete mixing) state is observed in the discharge with higher recycling. The increase of the hydrogen fraction in the core $\left(r_{\text {eff }} / a_{99}=0.68\right)$ is smaller even for the same beam fueling. It is interesting that the hydrogen fraction near the plasma edge $\left(r_{\text {eff }} / a_{99}=0.96\right)$ decreases for both discharges.

The density fluctuation spectrum shows a clear difference between these two discharges. The density fluctuation is peaked at the lower frequency $(f<40 \mathrm{kHz})$ regime in the plasma with isotope mixing state. In contrast, the density fluctuation is peaked at the higher frequency (40 $\mathrm{kHz}<f<90 \mathrm{kHz}$ ) regime in the plasma with isotope 
non-mixing state. It should be noted that the higher frequency peak appears at the ECH phase both in these two discharges. The ratio of density fluctuation amplitude integrated in the range of $40-90 \mathrm{kHz}$ to that of $4-40$ $\mathrm{kHz}$ decreases significantly after the switch from ECH to NBI heating The ratio stays low in the discharge with isotope mixing state, but starts to increase to the level in ECH phase in the discharge with isotope non-mixing state. These results also show the strong correlation between isotope mixing and density turbulence spectrum.

The difference of recycling results in the difference in the density profile. The density profile becomes flat for the discharge with lower recycling, while it becomes hollow in the discharge with higher recycling. Figure 6 shows the radial proles of electron density, hydrogen density, deuterium density, fraction of hydrogen density $n_{\mathrm{H}} /\left(n_{\mathrm{H}}+n_{\mathrm{D}}\right)$, and radial electric field at the time for the same line-averaged electron density. In the discharge with non-mixing state, the electron density is flat and density gradient in the region of $r_{\text {eff }} / a_{99}=0.68-0.86$ is negative. In contrast, the electron density is flat and the density gradient in the region of $r_{\text {eff }} / a_{99}=0.68-0.86$ is positive in the discharge with mixing state. Because of the beam fueling of hydrogen beam, the hydrogen density in the isotope mixing state is lower than the hydrogen density in the non-mixing state. In contrast, the deuterium density in the isotope mixing state is higher than the deuterium density in the non-mixing state due to the higher influx of deuterium as the exchange of hydrogen outflux to the wall. There are clear differences in the gradient of hydrogen fraction. The gradient of hydrogen fraction of that region in the discharge with isotope nonmixing is much larger than that in the discharge with isotope mixing even for the same line-averaged density and hydrogen beam fueling rate. The radial profiles of radial electric field are almost identical between the discharges with isotope mixing and non-mixing. The density fluctuation spectrum shows clear peak at $60 \mathrm{kHz}$ in the isotope non-mixing plasma. This peak disappears in the isotope mixing plasma. This observation demonstrates that the sign of the density gradient has a strong impact on the isotope mixing turbulence characteristics. Linear gyrokinetic simulation predicts stronger decrease of growth rate for TEM than that for ITG turbulence [39]. The transition from TEM turbulence to ITG turbulence is expected by changing the sign of the density gradient from negative to positive [39].

\section{ISOTOPE MIXING TRIGGERED BY PELLET INJECTION}

The shallow pellet injection [40] is a useful tool to trigger the transition from isotope non-mixing state to isotope mixing state, because the particles deposited near the plasma periphery at $r_{\text {eff }} / a_{99} \sim 0.9$ results in both the increase of collisionality and hollow density profile, in which the electron density gradient is positive. The

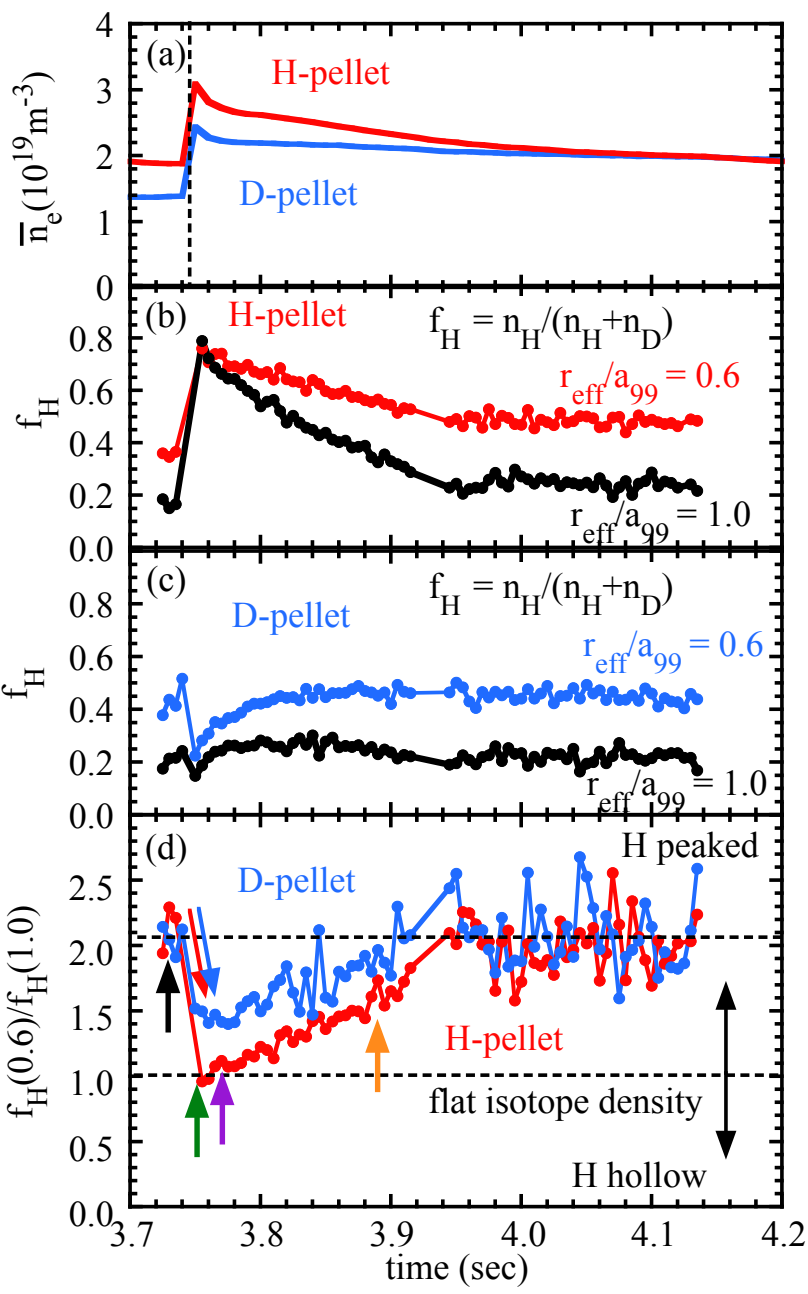

FIG. 7: Time evolution of (a) line averaged electron density, (b) (c) the fraction of hydrogen density $f_{\mathrm{H}}=\left[n_{\mathrm{H}} /\left(n_{\mathrm{H}}+n_{\mathrm{D}}\right)\right]$ at $r_{\text {eff }} / a_{99}=0.6$ and 1.0, respectively, and (d) the ratio of hydrogen fraction at $r_{\text {eff }} / a_{99}=0.6$ to that at $r_{\text {eff }} / a_{99}=1.0$ in the hydrogen and deuterium mixture plasma with hydrogen beam fueling and with hydrogen $(\mathrm{H})$ pellet injection (\#142315) or deuterium (D) pellet injection (\#142314).

hydrogen and deuterium pellet injection experiments are performed to study the time scale of isotope mixing. Hydrogen and deuterium pellet is injected to the isotope mixture plasma with hydrogen beam fueling and with deuterium recycling, where the hydrogen fraction is peaked in non-mixing state. If the plasma remains in the non-mixing state, the hydrogen pellet causes the flattening of hydrogen fraction, while the deuterium pellet causes the peaking of hydrogen fraction. However, when the transition from non-mixing state to mixing state occurs, the hydrogen fraction profile becomes flat regardless of the species of the pellet.

Figure 7 shows line averaged electron density, the fraction of hydrogen density $f_{\mathrm{H}}=\left[n_{\mathrm{H}} /\left(n_{\mathrm{H}}+n_{\mathrm{D}}\right)\right]$ at $r_{\mathrm{eff}} / a_{99}$ 
$=0.6$ and 1.0, respectively, and the ratio of hydrogen fraction at $r_{\text {eff }} / a_{99}=0.6$ to that at $r_{\text {eff }} / a_{99}=1.0$ in the hydrogen and deuterium mixture plasma with hydrogen beam fueling and with hydrogen pellet injection or deuterium pellet injection. Associated with the pellet injection, the line averaged electron density sharply increase by $\sim 50 \%$. Here the decay of hydrogen fraction rather than the decay of ion density in pellet is discussed. The decay of the ion density indicates the transport characteristics which are related to the so-called diffusion coefficient. It was reported that the decay time of hydrogen density after the hydrogen pellet is almost identical to the decay time of deuterium after the deuterium pellet [2]. This result demonstrates that the diffusion coefficient of hydrogen is similar to that of deuterium $\left(D_{\mathrm{H}} \sim D_{\mathrm{D}}\right)$. The decay of the isotope fraction is determined by the combination of decay of the species injected by pellet and recycling. For example, when the deuterium wall recycling is dominant, the decay of hydrogen fraction at the plasma edge $\left(r_{\text {eff }} / a_{99}=1.0\right)$ after the hydrogen pellet is determined by the exchange of species with the outward hydrogen flux and inward deuterium flux. In contrast, in the discharge with deuterium pellet, the increase of hydrogen fraction due to the decrease of deuterium density at the plasma edge is simply determined by the edge deuterium particle confinement time with the recycling ratio of unity where the outward deuterium flux is balanced with the inward deuterium flux.

Hydrogen fraction $f_{\mathrm{H}}$ both in the plasma core at $r_{\text {eff }} / a_{99}=0.6$ and plasma boundary at $r_{\text {eff }} / a_{99} 1.0$ increase after the hydrogen pellet and gradually decreases in $0.2 \mathrm{sec}$. In contrast, the hydrogen fraction $f_{\mathrm{H}}$ at $r_{\text {eff }} / a_{99}=0.6$ and 1.0 decreases after the deuterium pellet and recovers in $0.1 \mathrm{sec}$. The time evolution of the fraction ratio at two radii, $f_{\mathrm{H}}\left(r_{\text {eff }} / a_{99}=0.6\right) / f_{\mathrm{H}}\left(r_{\text {eff }} / a_{99}=1.0\right)$, is plotted as an indication of hydrogen peaking. This hydrogen peaking parameter decreases after the pellet injection of both hydrogen and deuterium, which indicates that the isotope mixing occurs by the pellet injection regardless of pellet species. Hydrogen peaking parameter for deuterium pellet is larger than that for hydrogen pellet, because the flattening of hydrogen fraction of the isotope mixing is partially canceled by the increase of deuterium ions near the plasma edge due to deuterium pellet fueling. Hydrogen peaking parameter gradually increases after the sharp drop and recovers to the level before the pellet injection within $0.2 \mathrm{sec}$. This indicates that the isotope mixing state after the pellet injection is transient (short period) and the plasma returns to the non-mixing state.

The radial profile of hydrogen fraction $15 \mathrm{~ms}$ before the pellet injection (dashed lines) and $5 \mathrm{~ms}, 25 \mathrm{~ms}$, and $145 \mathrm{~ms}$ after the hydrogen $(\mathrm{H})$ and deuterium $(\mathrm{D})$ pellet injection are plotted in Figure 8 . The increment of electron density by pellet injection is evaluated from the difference in density profile before and after the pellet injection, which indicates the source profile due to pellet injection. The maximum increment of electron density is located
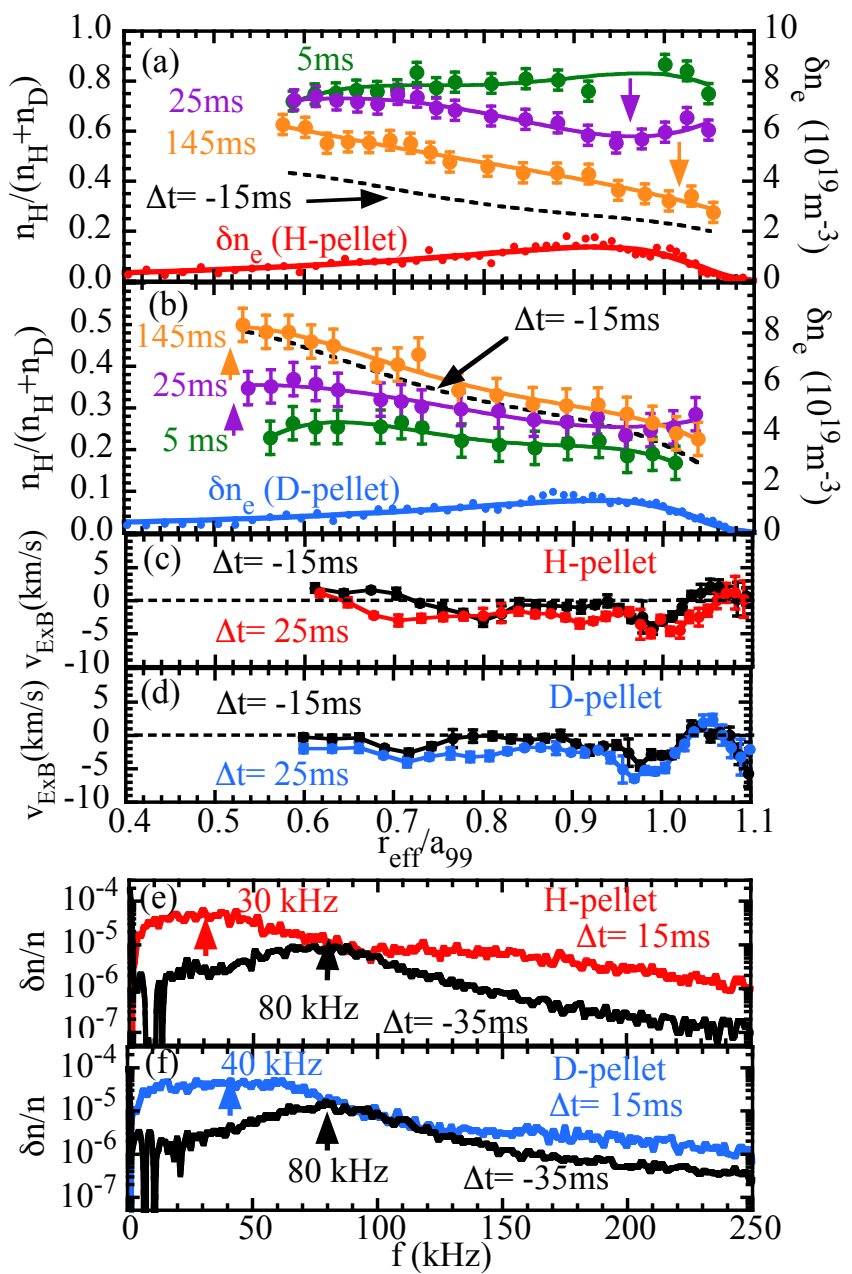

FIG. 8: Radial profiles of (a)(b) fraction of hydrogen density $n_{\mathrm{H}} /\left(n_{\mathrm{H}}+n_{\mathrm{D}}\right)$, and (c)(d) $E \times B$ flow velocity, and (e)(f) density fluctuation spectrum before $(\Delta t<0)$ and after $(\Delta t>0)$ (a)(c)(e) the hydrogen $(\mathrm{H})$ pellet injection (\#142315) and (b)(d)(f) the deuterium (D) pellet injection (\#142314). The radial profiles of the increment of electron density $\delta n_{e}$ by pellet injection are also plotted.

at $r_{\text {eff } /} a_{99}=0.95$ because of the shallow pellet penetration in this plasma. Before the pellet injection, the hydrogen fraction is peaked because of the beam fueling of hydrogen NBI in both cases. After the hydrogen pellet injection, the hydrogen fraction increases in the level of 0.8 with a flat profile. The flattening of hydrogen fraction is due to the hydrogen fueling near the plasma edge by hydrogen pellet and isotope mixing. At $25 \mathrm{~ms}$ after the pellet injection, the hydrogen fraction profile starts to be peaked due to the decay of hydrogen near the plasma edge injection by pellet. Further hydrogen peaking occurs due to the back transition from mixing state to non-mixing state. Then the gradient of hydrogen fraction at $145 \mathrm{~ms}$ after the injection becomes comparable to that before the pellet injection, although the absolute 
value of hydrogen fraction is still higher before the pellet injection.

In the discharge for deuterium pellet injection, the hydrogen fraction decreases to 0.2 and the hydrogen fraction profile becomes flat. Because the deuterium fueling by the pellet is mainly near the edge as seen in the radial profile of the increment of electron density, this is clear evidence for the isotope mixing. The hydrogen fraction starts to increase $25 \mathrm{~ms}$ after the pellet injection due to the decay of deuterium and the hydrogen fraction profile becomes almost identical to that before the pellet injection at $145 \mathrm{~ms}$ after. These results demonstrate that the flattening of hydrogen fraction is observed immediately (at $5 \mathrm{~ms}$ ) after the pellet injection. This rapid flattening indicates that the large ion diffusion coefficient (i.e., $\left.D_{i} / D_{e} \gg 1\right)$ which is consistent with the prediction of simulation for ITG turbulence. On the other hand, the recovery of the peaked hydrogen fraction after the pellet injection is relatively slow $(\sim 100 \mathrm{~ms})$ and this slow recovery indicates the small ion diffusion (i.e., $D_{i} / D_{e} \ll 1$ ) which is consistent with the prediction of simulation for TEM turbulence.

As seen in the radial profiles of $E \times B$ flow velocity, the radial electric field is close to zero in the core and becomes more negative (in the electron diamagnetic direction) near the plasma edge $\left(r_{\text {eff }} / a_{99}<1\right)$ and positive (in the ion diamagnetic direction) outside the plasma boundary $\left(r_{\text {eff }} / a_{99}>1\right)$. The $E \times B$ flow velocity increases slightly in the electron diamagnetic direction in the core after the pellet injection. The density fluctuation spectrum has a clear peak at $80 \mathrm{kHz}$ before the pellet injection $(\Delta t=-35 \mathrm{~ms})$. This is quite similar to the spectrum in the plasma with higher collisionality (Fig.1), higher $T_{e} / T_{i}$ ratio (Fig.3), and flat density profile (Fig.4). After the pellet injection, the peak at $80 \mathrm{kHz}$ disappears and increase of density fluctuation at lower frequency (20-60 $\mathrm{kHz}$ ) becomes more significant. The density fluctuation spectrum after the pellet injection $(\Delta t=15 \mathrm{~ms})$ is quite similar to that isotope mixing plasma in higher collisionality plotted in Figure 2.

The short period of mixing state is due to the quick recovery of $T_{e} / T_{i}$ ratio and density profile. Figure 9 shows time evolution of electron density, (b) electron and ion temperature in $r_{\text {eff }} / a_{99}=0.87$. After the pellet injection, the $T_{e} / T_{i}$ ratio drops to unity but the ratio recovers quickly $(\sim 50 \mathrm{~ms})$ to the level before the pellet injection. As seen in time evolution of electron density at $r_{\text {eff }} / a_{99}=0.68$ and 0.87 in Figure $9(\mathrm{a})$, the density gradient at $r_{\text {eff }} / a_{99}=0.68-0.87$ is negative before the pellet injection. After the pellet injection, the density gradient becomes positive until $t=3.84 \mathrm{sec}$. Although the decay of the electron density is slow $(\sim 0.3 \mathrm{sec})$, the change of the sign of density gradient at $r_{\text {eff }} / a_{99}=0.68-0.86$ is relatively short $(\sim 0.1 \mathrm{sec})$. After the pellet injection, the density fluctuation amplitude integrated in the range of 10-80 kHz increases sharply but its amplitude quickly decays $(\sim 50 \mathrm{~ms})$ to the levels before the pellet injection. The frequency spectrum of the density fluctuation
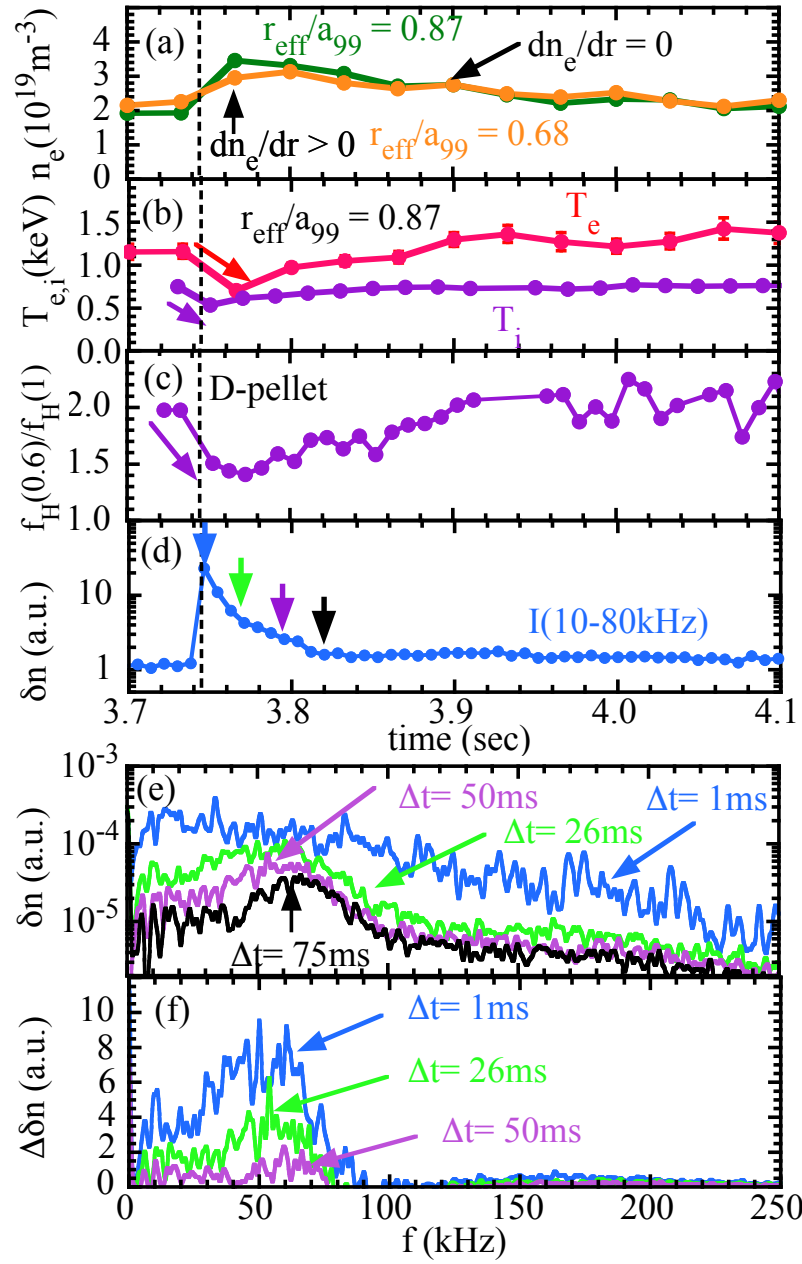

FIG. 9: Time evolution of (a) electron density at $r_{\text {eff }} / a_{99}=$ 0.68 and 0.87 , (b) electron and ion temperature at $r_{\mathrm{eff}} / a_{99}=$ $0.87,(\mathrm{c})$ the ratio of hydrogen fraction $f_{\mathrm{H}}=\left[n_{\mathrm{H}} /\left(n_{\mathrm{H}}+n_{\mathrm{D}}\right)\right]$ at $r_{\text {eff }} / a_{99}=0.6$ to that at $r_{\text {eff }} / a_{99}=1.0$, and (d) density fluctuation amplitude integrated in the range of $10-80 \mathrm{kHz}$, and frequency spectrum of (e) density fluctuation and (f) increment of density fluctuation after the pellet injection in the hydrogen and deuterium mixture plasma with hydrogen beam fueling and with deuterium (D) pellet injection (\#142314).

and increment of density fluctuation after the pellet injection are also plotted. Here the increment of density fluctuation by pellet injection is defined as $\Delta \delta n(\Delta t)=$ $\delta n(\Delta t)-\delta n(\Delta t=-35 \mathrm{~ms})$. The increase of density fluctuation is mainly in the flow frequency range below the peak of density fluctuation $(80 \mathrm{kHz})$ observed in non-mixing state in Figure 8 . The spectrum of density fluctuation $50 \mathrm{~ms}$ after the pellet injection is almost identical to that before the pellet injection and the increment becomes small, which indicates that the turbulence increases by pellet is transient. These characteristics of density turbulence are consistent with the short period of mixing 
state after the pellet injection. Time evolution of hydrogen peaking factor drops from 2.0 to 1.5 after the pellet injection. Then the peaking factor remains at low level for a short time $(\sim 50 \mathrm{~ms})$ then starts to increase from $t=3.8$ secs. The time evolution of density fluctuation and hydrogen peaking factor strongly suggests the linkage of turbulence and isotope mixing as predicted by the gyrokinetic simulation.

\section{DISCUSSION AND SUMMARY}

Typical characteristics of plasma with isotope nonmixing and mixing state plasmas are summarized in Table 1 .

Table 1: Typical characteristics of plasma with isotope non-mixing and mixing state plasmas.

\begin{tabular}{|c||c|c|}
\hline parameters & non-mixing state & mixing state \\
\hline isotope density ratio & non-uniform & uniform \\
\hline electron density & $<2-3 \times 10^{19} \mathrm{~m}^{-3}$ & $>2-3 \times 10^{19} \mathrm{~m}^{-3}$ \\
\hline density gradient & $d n_{e} / d r \leq 0$ & $d n_{e} / d r>0$ \\
\hline$T_{e} / T_{i}$ ratio & large $(>1-2)$ & small $(<1-2)$ \\
\hline peak frequency & $60-90 \mathrm{kHz}$ & $20-40 \mathrm{kHz}$ \\
\hline intrinsic toroidal flow & co-direction & counter-direction \\
\hline
\end{tabular}

The plasma parameters preferable for the non-mixing state are 1) lower collisionality, 2) higher $T_{e} / T_{i}$ and higher $\left.\left(R / L_{T_{e}}\right) /\left(R / L_{T_{i}}\right), 3\right)$ negative or zero density gradient (peaked or flat density profile), where the TEM is expected to be destabilized. In LHD, first and second preferable plasma parameters (low collisionality and higher $\left.T_{e} / T_{i}\right)$ can be often obtained in the low density plasma with ECH and with negative NBI where the beam energy is high enough $(160-180 \mathrm{keV})$ to heat electrons rather than ions. The preferable plasma parameters for mixing, that is higher collisionality and lower $T_{e} / T_{i} \sim 1$, and positive density gradient (hollow profile), are produced by shallow pellet injection. The peaking of isotope species of beam fueling is not always observed in the isotope mixture plasma with non-mixing state. In order to observe the peaking of beam fueled isotope species, the number of particles of the species supplied by the beamfueling in the core should be large enough compared with the number of particles supplied by recycling and gas puff. For example, when the species of bean is hydrogen, the hydrogen recycling should be significantly reduced, that is $\Gamma_{\mathrm{H}} / \Gamma_{\mathrm{D}}<1$. In the case of higher hydrogen recycling $\left(\Gamma_{\mathrm{H}} / \Gamma_{\mathrm{D}}>1\right)$, the peaking of hydrogen fraction can not be achieved even in the isotope mixture plasma with non-mixing state. The conditions for observing the peaking of beam fueled isotope species are 1) isotope mixture plasma, 2) non-mixing state $\left(D_{i} / D_{e} \ll 1\right)$, low recycling of beam fueling $\left(\Gamma_{\text {beam-species }} / \Gamma_{\text {recycling-species }}<1\right)$.

The strong correlations between isotope mixing and turbulence characteristics are observed in LHD. The peaking of beam fueled species is a direct indication of non-mixing but can be observed only in the condition of low recycling of beam species, which are not always achieved. In contrast, the turbulence characteristics are found to be relating to the isotope mixing and nonmixing. The density fluctuation peak at $80 \mathrm{kHz}$ is observed in the plasma with non-mixing state, while this peak disappears in the plasma with mixing state. This is a useful finding, because the turbulence characteristics can be obtained even in the condition of higher recycling of beam species. Furthermore, the reversal of intrinsic toroidal flow also suggests the change in the turbulence characteristics because the direction of intrinsic toroidal flow driven by residual stress strongly depends on the turbulence mode (TEM or ITG), which has been observed widely in tokamaks. It is difficult to identify the turbulence mode (for example, TEM or ITG) from the spectrum of density fluctuation. However, significant differences in density fluctuation spectrum observed between isotope mixing and non-mixing states demonstrate that the change in turbulence observed is a strong candidate for the mechanism causing the transition between isotope mixing and non-mixing state.

Microinstability characteristics for the plasma with isotope non-mixing and mixing state are identified by the linear gyrokinetic calculations, and are summarized in the Table 2. In LHD, the TEM can be destabilized near the plasma edge due to the large density gradient and ITG mode or TEM mode can be destabilized in the core region. In this experiment, the ITG mode in the core region is destabilized for both non-mixing and mixing state. The TEM is destabilized in the non-mixing state and stabilized in mixing state, which suggests that the TEM contributes the non-mixing state, where the $D_{e} \gg D_{i}$ is expected [11].

Table 2: Linear gyrokinetic simulation results for the plasma with isotope non-mixing and mixing state

\begin{tabular}{|c||c|c|}
\hline & non-mixing state & mixing state \\
\hline edge $r_{\text {eff }} / a_{99}=0.9$ & TEM destabilized & TEM stabilized \\
\hline core $r_{\text {eff }} / a_{99}=0.5$ & ITG destabilized & ITG destabilized \\
\hline
\end{tabular}

The dependence of plasma parameters (collisionality, $T_{e} / T_{i}$ ratio, density gradient) for the transition from nonmixing to mixing transition is consistent with plasma parameter dependence where the gyrokinetic simulation predicts the transition from TEM to ITG in the plasma core. These experimental observations in LHD are in contrast to the observation of isotope mixing state in JET where the large ion diffusion is observed in the isotope mixture ( $\mathrm{H}$ and $\mathrm{D})$ plasma. In JET experiment, the electron density is higher $\left(\bar{n}_{e} \sim 3 \times 10^{19} \mathrm{~m}^{-3}\right)$ and the $T_{e} / T_{i}$ is close to unity and ITG turbulence is expected to be dominant [41]). The isotope mixture experiment in the low density and high $T_{e} / T_{i}$ plasma in tokamak would be interesting. This is because the comparison of characteristics of the plasma with non-mixing state be- 
tween tokamak and helical plasma provides the deeper understanding of isotope mixing mechanism, which is indispensable for the better control of $\mathrm{D}-\mathrm{T}$ isotope mixture plasma in future.

\section{ACKNOWLEDGEMENTS}

The authors are grateful to the technical staff of LHD for their excellent support for this work. One of the au- thors (KI) acknowledges Dr. M.Yoshida (National Institutes for Quantum and Radiological Science and Technology; QST) for providing us two spectrometers for the bulk charge exchange spectroscopy. This work is supported by the National Institute for Fusion Science grant administrative budgets (NIFS10ULHH021) and JSPS KAKENHI Grant Numbers JP15H02336, JP16H02442, JP17H01368, and JP17K14899.
[1] Ida, K., Adv. Phys. X 5, 1801354 (2020).

[2] Ida, K., et al. Nucl. Fusion 59, 056029 (2019).

[3] Bourdelle, C., et al. Phys. Plasmas 14, 112501 (2007).

[4] Citrin, J., et al. Plasma Phys. Control. Fusion 59, 124005 (2017).

[5] Peeters A.G., et al. Comput. Phys. Commun. 180, 2650 (2009).

[6] Bourdelle, C., et al. Nucl. Fusion 58, 076028 (2018).

[7] Adam, J.C, Tang, W.M., Rutherford, P.H., Phys. Fluids 19, 561 (1976).

[8] Dannert, T., and Jenko, F., Phys. Plasmas 12, 072309 (2005).

[9] Romanelli, F., Phys. Fluids B 1, 1018 (1989).

[10] Rosenbluth, M.N., and Hinton, F.L., Phys. Rev. Lett. 80, 824 (1998).

[11] Ida, K., et al. Phys. Rev. Lett. 124, 025002 (2020).

[12] Watanabe, T.-H., et al. Nucl. Fusion 46, 24 (2006).

[13] Nunami, M., et al. Phys. Plasmas 19, 042504 (2012).

[14] Ishizawa, A., et al. Nucl. Fusion 55, 043024 (2015).

[15] Nakata, M., et al. Plasma Phys. Control. Fusion 58, 074008 (2016).

[16] Nunami, M., et al. Plasma Phys. Control. Fusion 59, 044013 (2017).

[17] Tanaka, K., et al. Plasma. Fusion. Res. 5, S2053 (2010).

[18] Tanaka, K., et al. Nucl. Fusion 57, 116005 (2017).

[19] Tanaka, K., et al. Nucl. Fusion 59. 126040 (2019).

[20] Tanaka, K., et al. Plasma Phys. Control. Fusion 62, 024006 (2020).

[21] Yamazaki, K., et al. Plasma. Fusion. Res. 13, 1202103 (2018).

[22] Haskey, S.R., et al. Rev. Sci. Instrum. 89, $10 \mathrm{D} 110$ (2018).

[23] Haskey, S.R., et al. Plasma Phys. Control. Fusion 60, 105001 (2018).

[24] Ida, K., et al. Rev. Sci. Instrum. 90, 093503 (2019).

[25] Tanaka, K., et al. Nucl. Fusion 48, 039801 (2008).

[26] Cao, M.N., et al. Nucl. Fusion 59, 104001 (2019).

[27] Nakata, M., et al. Phys. Rev. Lett. 118, 165002 (2017).

[28] Tanaka, K., et al. Rev. Sci. Instrum. 79, $10 \mathrm{E} 702$ (2008).

[29] Michael, C. A., et al. Rev. Sci. Instrum. 86, 093503 (2015).

[30] Zhong, W.L, Phys. Plasmas 23, 014037 (2016).

[31] Lee, J.A., Phys. Plasmas 25, 022513 (2018).

[32] Arnichand, H, et al. Plasma Phys. Control. Fusion 58, 014037 (2016).

[33] Nunami, M., et al. Plasma. Fusion. Res. 6, 1403001 (2011).

[34] Kasuya, N., et al. Nucl. Fusion 58, 106033 (2018).
[35] Diamond, P.H., et al. Nucl. Fusion 49, 045002 (2009).

[36] Ida, K., et al. Nucl. Fusion 54, 045001 (2014).

[37] Nagaoka, K., et al. Phys. Plasmas 20, 056116 (2013).

[38] Rice, J.E., et al. Phys. Rev. Lett. 107, 265001 (2011).

[39] Nakata, M., et al. Plasma Phys. Control. Fusion 61, 014016 (2019).

[40] Sakamoto, R., et al. Nucl. Fusion 41, 381 (2001).

[41] Maslov, M., et al. Nucl. Fusion 58, 076022 (2018). 